\title{
Irreversible transition of amorphous and polycrystalline colloidal solids under cyclic deformation
}

\author{
Pritam Kumar Jana, ${ }^{1,2, *}$ Mikko J. Alava, ${ }^{1}$ and Stefano Zapperi ${ }^{1,3,4}$ \\ ${ }^{1}$ COMP Centre of Excellence, Department of Applied Physics, Aalto University, P.O. Box 11100, FI-00076 Aalto, Espoo, Finland \\ ${ }^{2}$ Université Libre de Bruxelles (ULB), Interdisciplinary Center for Nonlinear Phenomena and Complex Systems, Campus Plaine, CP 231, \\ Blvd. du Triomphe, B-1050 Brussels, Belgium \\ ${ }^{3}$ Center for Complexity and Biosystems, Department of Physics, University of Milano, via Celoria 16, 20133 Milano, Italy \\ ${ }^{4}$ CNR-ICMATE, Via R. Cozzi 53, 20125, Milano, Italy
}

(Received 26 June 2018; published 14 December 2018)

\begin{abstract}
Cyclic loading on granular packings and amorphous media leads to a transition from reversible elastic behavior to an irreversible plasticity. In the present study, we investigate the effect of oscillatory shear on polycrystalline and amorphous colloidal solids by performing molecular dynamics simulations. Our results show that close to the transition, both systems exhibit enhanced particle mobility, hysteresis, and rheological loss of rigidity. However, the rheological response shows a sharper transition in the case of the polycrystalline sample as compared to the amorphous solid. In the polycrystalline system, we see the disappearance of disclinations, which leads to the formation of a monocrystalline system, whereas the amorphous system hardly shows any ordering. After the threshold strain amplitude, as we increase the strain amplitude both systems get fluid. In addition to that, particle displacements are more homogeneous in the case of polycrystalline systems as compared to the amorphous solid, mainly when the strain amplitude is larger than the threshold value. We do not see any effect of oscillation frequency on the reversible-irreversible transition.
\end{abstract}

DOI: 10.1103/PhysRevE.98.062607

\section{INTRODUCTION}

A common characteristic of a number of soft materials is that when such materials are subjected to a small shear stress they behave elastically and reversibly, while they display plastic behavior when they are subjected to a high shear stress [1]. A similar behavior is also exhibited by periodically driven disordered granular media [2-4], colloidal suspensions [5-8], and colloidal gels [9]. A number of simulations and experiments have shown that depending on the oscillatory strain amplitude they display an intriguing transition from reversible elastic behavior to irreversible plastic deformation [10-16].

Soft glassy materials, such as foams, concentrated colloids, pastes, and clays are viscoelastic materials. Under mechanical perturbation, their response is time and amplitude dependent [17]. When a small stress or strain is applied, they exhibit solidlike behavior on short timescales but on much longer timescales they start to flow like liquids. In a similar way when the strain amplitude is varied, a transition from mostly solidlike to mostly liquidlike is observed. To characterize the rheological properties of those soft systems, an oscillatory perturbation is among the most commonly used tools as the variation of driving strain amplitudes allows quantifying the elastic, viscous, and plastic behavior in materials [18]. At small deformations, where systems exhibit elastic behavior, storage modulus dominates, whereas at large deformations, where systems display irreversible plastic behavior, loss modulus plays a dominating role instead [19].

\footnotetext{
*pritam.jana@aalto.fi
}

The microscopic features corresponding to the macroscopic rheological response in small and large strain amplitude regimes is a matter of discussions. For crystalline materials, it is well established that yielding is mediated by defects. For amorphous solid, it is considered that localized rearrangements, known as shear transformations, are responsible for flow [17]. However, it is challenging to identify those local events due to their disordered structure. In between those two classes of materials, crystals and amorphous solids, lie polycrystals, where several crystalline regions are separated by grain boundaries. Plastic deformation of colloidal polycrystals has been studied in experiments and in simulations [20-22]. In a previous study, we have shown that polycrystals also exhibit yielding transition mediated via defects motion depending on the strain amplitude of oscillatory shear [23]. There are few studies [24,25] comparing polycrystals and amorphous solids, though this could shed light on the nature of yielding transition.

In the present study, we approach the irreversibility transition under oscillatory shear by comparing $2 \mathrm{D}$ amorphous solid and polycrystalline colloidal systems by performing molecular dynamics simulations. Our results show that the rheological response for both systems is similar before and after the transition, i.e., at a low strain amplitude, the storage modulus dominates over the loss modulus, and the difference decreases when the strain amplitude crosses a threshold value. However, the irreversible transition is sharper in the case of the polycrystalline sample as compared to the amorphous solid. A similar structural change is observed in radial distribution functions, though the effect is much weaker in amorphous solids. Particle displacements in polycrystalline samples is more homogeneous as compared to amorphous 
solid. We also show the atomic strain close to the transition for both cases, which reveals the sliding of the dislocations through the crystals and grain boundary motion and finally annihilation of the dislocations, whereas amorphous solids exhibit local rearrangements with no annihilation of defects. We discuss the effect of oscillation frequencies on the irreversible transition. We also briefly shed light on the thermal effect on the irreversible transition. This paper is organized as follows: in Sec. II we describe the model and simulation details; the results and discussions are presented in Sec. III; the conclusions are presented in Sec. IV.

\section{MODEL AND SIMULATION DETAILS}

Molecular dynamics simulations are performed on 2D amorphous and polycrystalline colloidal systems. Both systems consist of bidisperse particles where the ratio of larger $(l)$ and smaller $(s)$ particles is 65:35 in amorphous solids [26] and that ratio is 0.5:99.5 for polycrystalline systems. We model attractive colloids as Lennard-Jones particles [27]. Two types of atoms, $\alpha, \beta=l$, s, which interact via LJ potential as follows:

$$
V_{\alpha, \beta}=4 \epsilon_{\alpha, \beta}\left[\left(\frac{\sigma_{\alpha, \beta}}{r}\right)^{12}-\left(\frac{\sigma_{\alpha, \beta}}{r}\right)^{6}\right]-C_{\alpha, \beta} .
$$

For amorphous solid the depths of the potential well $\epsilon_{\alpha, \beta}$ are $\epsilon_{l l}=1.0, \epsilon_{l s}=1.5, \epsilon_{s s}=0.5$. $\sigma_{\alpha, \beta}$ is the finite distance at which interparticle $(\alpha, \beta)$ potential becomes zero which takes the value $\sigma_{l l}=1.0, \sigma_{s s}=0.88$, and $\sigma_{l s}=0.8$. The cutoff distance $r_{c}^{\alpha, \beta}$ is fixed to $2.5 \sigma_{\alpha, \beta}$. The same parameters for polycrystalline system are as follows: $\epsilon_{l l}=1.0, \epsilon_{s s}=0.5$, $\epsilon_{l s}=1.5 ; \sigma_{l l}=1.4, \sigma_{s s}=1.0, \sigma_{l s}=1.2$ and the cutoff radius is $r_{c}^{\alpha, \beta}=3.0 \sigma_{\alpha, \beta}$. Mass of the particles is $m_{l}=m_{s}=1$. The constant $C_{\alpha, \beta}$ ensures the continuity of $V_{\alpha, \beta}$ at $r=r_{c}^{\alpha, \beta}$. The unit of length, $\sigma$, is set to $\sigma_{l l}$ and $\sigma_{s s}$ for the amorphous and polycrystalline systems, respectively. The unit of mass and energy are set to $m=m_{l}$ and $\epsilon=\epsilon_{l l}$, respectively, and correspondingly the unit of time is $\tau$ defined as $\sigma \sqrt{(} \mathrm{m} / \epsilon)$. The equation of motion is integrated using the Verlet algorithm with the time step $\Delta t=0.005 \tau$.

The amorphous and polycrystalline systems are prepared as follows: first both systems are equilibrated at a sufficiently high temperature. The temperature is controlled by NoseHoover thermostat with the damping time of $0.1 \tau$. Then the system is gradually cooled down with a slow rate to the target temperature and then is relaxed for $5000 \tau$. The procedure is repeated for 10 independent samples. The packing fraction of the system is defined as $\phi=\pi\left[N_{l} R_{l}^{2}+N_{s} R_{s}^{2}\right] / L^{2}$ and is kept fixed at 0.79 and 0.84 for the polycrystalline and amorphous system, respectively.

The linear dimensions of amorphous and polycrystalline samples are $92.78 \times 92.78$ and $100 \times 100$, respectively. In the case of the polycrystalline system, several grains with local crystal orientations are separated by grain boundaries which consist of a regular array of dislocations. The combination of particles with five and seven neighbors known as positively charged $(+1)$ and negatively charged $(-1)$ disclination, respectively [28], create edge dislocations. Dislocations can exist individually or can form an array depending on the misorientation of the grains which is consistent with Frank condition, $n \propto \sin d \theta$, where $n$ is the line density along the grain boundary with the corresponding misorientaion $d \theta$ [29].

In a passing note, the grain boundaries are not pinned at both sides. They evolve with the atomic rearrangement. However, the presence of bigger particles makes the boundary stronger as we showed in our previous work [23]. In the case of an amorphous solid, there is no long-range ordering.

To discuss the temperature effect on irreversible transition for both cases, we prepare sample at $T=0.001,0.01,0.1,0.2$ for amorphous solid and at $T=0.001,0.01,0.1$ for polycrystalline sample. After quenching the sample to the target temperature with a similar cooling rate, we relax them for $205000 \tau$.

Periodic shear strain was applied in the $x y$ plane using LeeEdwards periodic boundary conditions. The shear strain was varied as a function of time according to

$$
\gamma(t)=\gamma_{0}(t) \sin \left(2 \pi t / T_{p}\right),
$$

where $\gamma_{0}$ is the strain amplitude and $T_{p}$ is the oscillation period. The corresponding oscillation frequency $\omega$ is $2 \pi / T_{p}$. The system was subjected to periodic deformation during 200 cycles, and the positions of all atoms were saved at the end of every cycle. The measured parameters are averaged over 10 independent simulations.

Molecular dynamics computation does not consider the particle-solvent interaction in contrast to Brownian dynamics. In the case of a densely packed system, particle-particle interactions guide the dynamics instead of the particle-solvent interactions [30]. There are several studies where colloidal systems are investigated by using molecular dynamics simulations [31-33]. Stevens et al. have investigated the shear melting of colloids by using molecular dynamics study [32]. Komatsugawa et al. [33] have constructed a molecular dynamics model to mimic the experiment where the behavior of a concentrated colloidal system is studied under oscillatory shear [34]. We choose to perform molecular dynamics simulations to study colloidal polycrystals and amorphous solid under cyclic deformation, and we use LAMMPS [35].

\section{RESULTS AND DISCUSSIONS}

\section{A. Enhanced mobility}

Depending on the strain amplitude, the local rearrangements of particles can be reversible after one or more cycles or irreversible, leading to chaotic dynamics and particle diffusion [36]. To characterize particle motion at $T=0.001$, we compute mean-square displacement (MSD) $\left\langle\Delta r^{2}\right\rangle$ at the end of each cycle $N_{c}$ considering the initial configuration $\left(N_{c}=0\right)$ as a reference. The MSD is defined as follows:

$$
\left\langle\Delta r^{2}\left(N_{c}\right)\right\rangle=\frac{1}{N}\left\langle\sum_{i=1}^{N}\left|\mathbf{r}_{i}\left(N_{c}\right)-\mathbf{r}_{i}(0)\right|^{2}\right\rangle,
$$

where $N$ is the number of particles. We plot the MSD as a function of the number of cycles for both amorphous and the polycrystalline systems in Fig. 1. The local slope averaged over the last 20 cycles is plotted as a function of strain amplitude $\gamma_{0}$ in Fig. 2. It shows that for lower values of strain amplitudes after an initial transient, the system becomes 


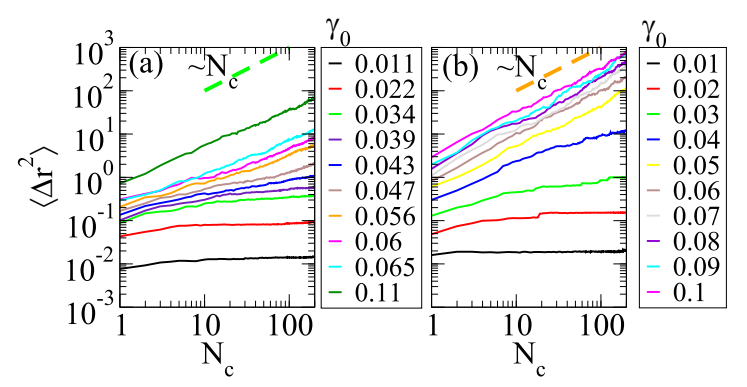

FIG. 1. MSD as a function of number of cycles, $N_{c}$, for (a) amorphous solid and (b) polycrystalline system for different values of $\gamma_{0}$. The smallest $\gamma_{0}$ is at the bottom of the figure and then gradually increases.

reversible. However, after a threshold value of $\gamma_{0}$, the system becomes diffusive within the simulation timescale. Both polycrystalline and amorphous solids respond in a similar way. It is important to note that for a similar value of strain amplitude, close to the threshold value and after the transition, MSD is larger in the case of a polycrystalline sample as compared to amorphous solid. The reason is that in the case of amorphous solid, rearrangements are local [37], whereas in the case of polycrystals whole grains (different local orientations) move coherently. This is also evident from the displacement distribution as shown in Fig. 7(f).

\section{B. Dynamic modulus}

To understand the hysteresis and the rheological loss of rigidity of the system, we compute storage modulus $G^{\prime}$ and loss modulus $G^{\prime \prime}[15,19]$. In purely elastic materials the stress and strain occur in phase, whereas in a purely viscous material strain lags stress by $90^{\circ}$. Viscoelastic materials exhibit behavior somewhere in between that of purely viscous and elastic materials [38]. In viscoelastic materials, storage and loss modulus are the measure of stored energy, representing the elastic contribution, and the dissipated energy, representing the viscous contribution, respectively. They are defined as

$$
\begin{aligned}
G^{\prime} & =\frac{\sigma_{0}^{x y}}{\gamma_{0}} \cos (\delta), \\
G^{\prime \prime} & =\frac{\sigma_{0}^{x y}}{\gamma_{0}} \sin (\delta),
\end{aligned}
$$

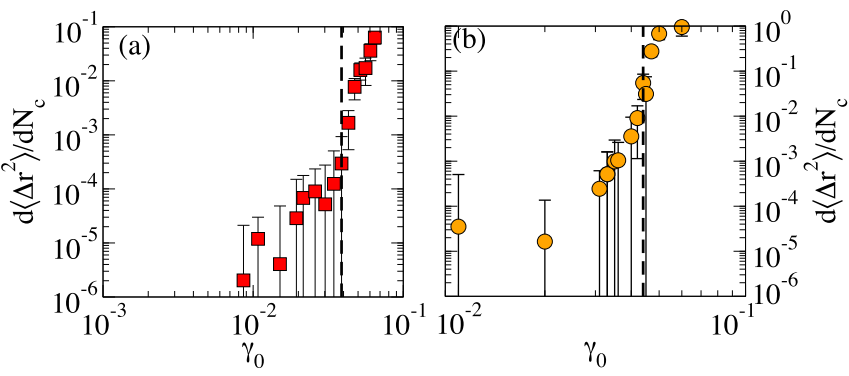

FIG. 2. Local slope of mean square displacement (MSD) vs. $N_{c}$ (see Fig. 1) is plotted as a function of strain amplitude at temperature $T=0.001$. (a) Amorphous solid and (b) polycrystals. The dotted lines indicate the strain amplitudes after which enhanced mobility is observed.

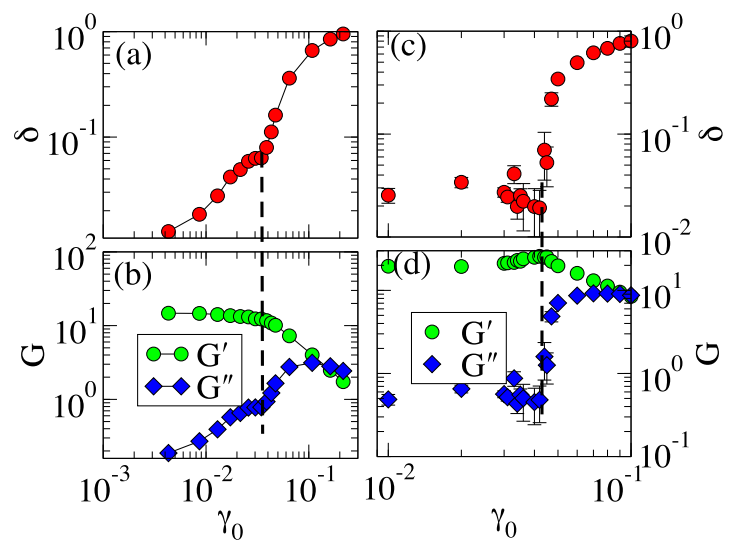

FIG. 3. (a), (c) Phase lag $\delta$; (b), (d) dynamic modulus $\left(G^{\prime}, G^{\prime \prime}\right)$ are shown as a function of $\gamma_{0}$ at temperature $T=0.001$ for amorphous solid (left) and for polycrystals (right).

where $\sigma_{0}^{x y}$ and $\delta$ are the shear stress amplitude, and the phase lag, respectively, obtained by fitting the shear stress-time plot with a oscillatory function $\sigma_{x y}=\sigma_{0}^{x y} \sin (\omega t+\delta)$ [39]. The ratio between shear loss and shear storage modulus in viscoelastic materials is defined as $\tan \delta$, which measures the dampening of material. We have plotted $G^{\prime}$ and $G^{\prime \prime}$ along with the phase lag as a function of $\gamma_{0}$ in Fig. 3. We observe that at a low value of $\gamma_{0}$, in both cases storage modulus $G^{\prime}$ dominates over loss modulus $G^{\prime \prime}$, which indicates that in the linear regime the response of both systems is like a soft elastic solid [19]. As we increase the strain amplitude, slow evolution of shear moduli is observed until $\gamma_{0}=0.0388$ and $\gamma_{0}=0.044$ for the amorphous and polycrystalline system, respectively, which indicates a certain degree of plastic rearrangements. Note that in the reversible regime, in the case of amorphous systems loss moduli and the phase lag $\delta$ weakly increase while polycrystalline systems exhibit a fluctuation. As $\gamma_{0}$ increases further, we observe a sharp increase of phase lag and the difference between storage and loss moduli significantly decreases, which indicates the onset of energy dissipation due to plastic deformation $[13,19]$. It is noteworthy that in the case of a polycrystalline system the transition from reversible to the irreversible regime is much sharper as compared to the amorphous solid. The reason is that in case of the polycrystalline system, as the $\gamma_{0}$ approaches the threshold value of strain amplitude, dislocations are annihilated, and the system becomes monocrystalline with defects due to the presence of bigger particles. In the case of an amorphous solid, no such phase change occurs. Therefore, a further increase of strain amplitude, a stronger defect-free monocrystalline sample starts to fluidize and exhibit a sharp transition as compared to the amorphous solid.

To understand the energy dissipation during the plastic events, one computes the hysteresis loop area from the shear stress against the applied strain. In our previous study, we have shown that the hysteresis loop area sharply increases as strain amplitude reaches a threshold value in case of a polycrystalline system [23]. In a similar study, Laurson et al. [40] have shown that cyclically stressed crystalline solids exhibit two phases: jammed and the moving state by computing hysteresis loop area. It is explained in the light of collective dislocation 

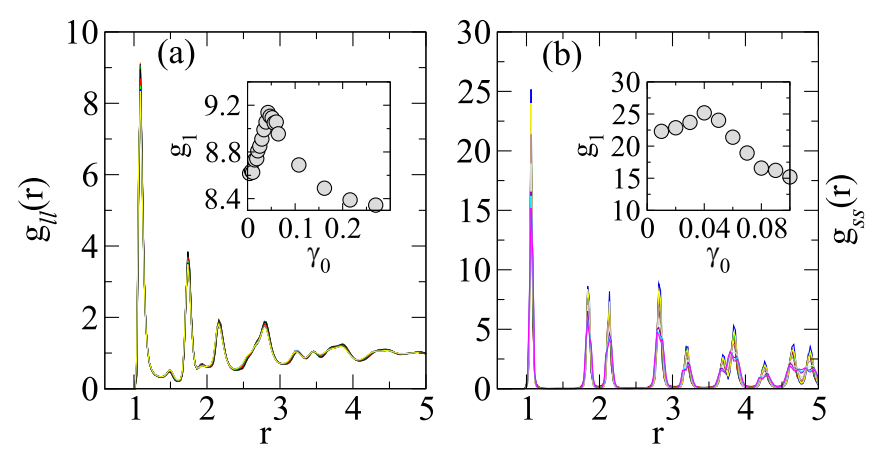

FIG. 4. Radial distribution function (RDF) at temperature $T=0.001$. Panel (a) corresponds to amorphous solid for different values of strain amplitude which include $\gamma_{0}=0.0043,0.009$, $0.011,0.015,0.019,0.022,0.026,0.03,0.034,0.039,0.043$, $0.047,0.052,0.056,0.06,0.065$. Panel (b) corresponds to polycrystals when the strain amplitudes are $\gamma_{0}=0.01,0.02,0.03$, $0.04,0.05,0.06,0.07,0.08,0.09,0.1$. Inset shows the first peak height of the RDF as a function of $\gamma_{0}$.

dynamics. Perchikov et al. have theoretically studied the response of amorphous solid under oscillatory shear where they have shown the presence of hysteresis after a threshold value [41]. In a recent simulation on amorphous solid under oscillatory shear, Leishangthem et al. have also shown the hysteresis after a threshold value of strain amplitude [12]. One can correlate the observation of hysteresis and the decrease of storage modulus after the threshold strain amplitude. Both are the results of energy dissipation due to plastic deformation.

\section{Structural changes}

To describe the local structure of the system depending on the shear strain amplitude, we compute the radial distribution function (RDF) as it quantifies the density variation as a function of the distance from a reference particle. It is mathematically defined as follows [26]:

$$
g_{l s}(r)=\frac{L^{2}}{2 \pi r N_{l} N_{s}} \sum_{i \in\{l\}} \sum_{j \in\{s\}}\left\langle\delta\left(r-r_{i j}\right)\right\rangle,
$$

where $N_{l}$ and $N_{s}$ are the number of particles of type $l$ (larger particles) and type $s$ (smaller particles), $L$ is the length of the system, and $r_{i j}$ is the distance between particle $i$ and $j$. For larger $r, g_{l s}(r)$ becomes 1.0. In Figs. 4(a) and 4(b) we have shown RDF of $l-l$ and $s$ - $s$ pairs for amorphous and polycrystalline systems, respectively. Inset figures display the height of the first peak obtained from RDF plot.

In the case of amorphous solids, we see an initial increase of the first peak height as a function of $\gamma_{0}$ until the same threshold value of $\gamma_{0}=0.0388$ after which the system exhibits enhanced mobility and rigidity loss. In the case of the amorphous solid, the height of the first peak corresponds to the density of the first neighbor of type $l$ (larger particles) around $l$-type (larger) particle. As the peak height increases, we conclude that there is a certain degree of rearrangement which leads to the increase of density of $l$-type particles around an $l$-type particle in amorphous solid. After the critical strain amplitude, the peak height of the radial distribution function decreases as we increase the strain amplitude. In the
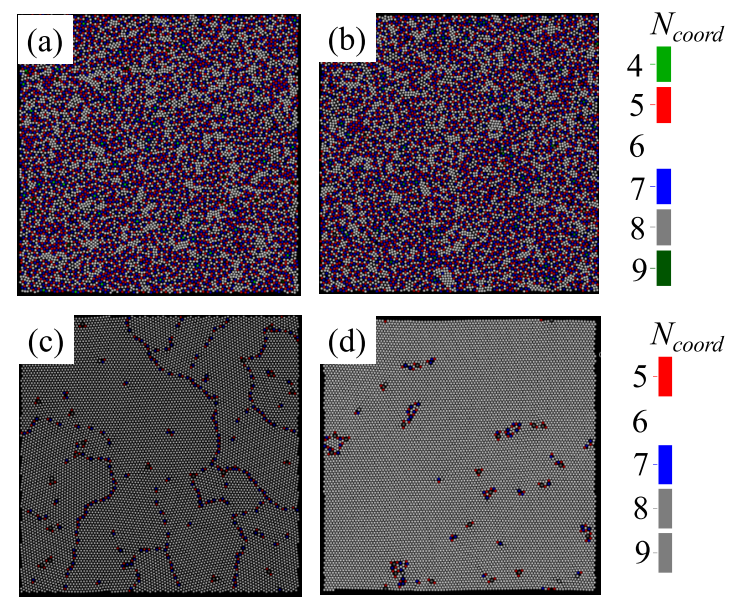

FIG. 5. Panels (a), (c) display the initial configuration of amorphous solid and polycrystalline system, respectively. Panels (b), (d) display the structures after 200 cycles for large values of strain amplitude which are $\gamma_{0}=0.22$ for amorphous solid and $\gamma_{0}=0.1$ for polycrystalline system. The color of each particle represents the number of neighbors $N_{\text {coord }}$. All are at temperature $T=0.001$.

case of polycrystalline systems, as in amorphous solids, as we increase the strain amplitude, the peak height in the radial distribution function increases until a threshold value which is a result of grain boundary motion and defect annihilation. After the threshold strain amplitude, both systems start to fluidize which is shown in the RDF plot where the first peak height exhibits a gradual decrease. To quantify the degree of order, we calculate $2 \mathrm{D}$ local bond-orientational order parameter $\psi_{6}$ for each particle $i$ as follows [42]:

$$
\psi_{6}\left(r_{i j}\right)=\frac{1}{n n} \sum_{j=1}^{n n} e^{i 6 \theta\left(\mathbf{r}_{\mathbf{i j}}\right)}
$$

where the summation is taken over all $n n$ nearest neighbors of the particle $i . \theta$ is the angle between the vector $\mathbf{r}_{\mathbf{i j}}$ connecting particle $i$ to $j$ and $x$ axis. In the case of a perfect hexagonal symmetry, $\left|\psi_{6}\right|=1$. Figure 6 shows the histogram of $\left|\psi_{6}\right|$ values of the particles. Figure 6(a) represents the amorphous solids and Fig. 6(b) is for the polycrystalline systems. In the case of amorphous solids, the maximum of the histogram is approximately 0.015 which is equivalent to the hexatic order parameter of isotropic liquids [42], and it remains unchanged with increasing the strain amplitude. $\left|\psi_{6}\right|>0.7$ indicates that the system is in a crystalline state $[42,43]$. In the case of a polycrystalline system, as we increase the strain amplitude, until a threshold value strain amplitude, the peak height of the histogram increases. A final configuration of the polycrystalline system under the oscillatory shear with a larger strain amplitude appears as a monocrystal, as depicted in Fig. 5(d).

To understand the fate of the defects for both systems under oscillatory shear, we have shown the structure of a polycrystalline and amorphous sample before and after the transition in Fig. 5. The color corresponds to the number of neighbors of each particle. The left panel in Fig. 5 [Figs. 5(a) and 5(c)] represents the initial configurations of an amorphous and a polycrystalline sample, respectively. We see mostly particles with five and seven neighbors in the amorphous 


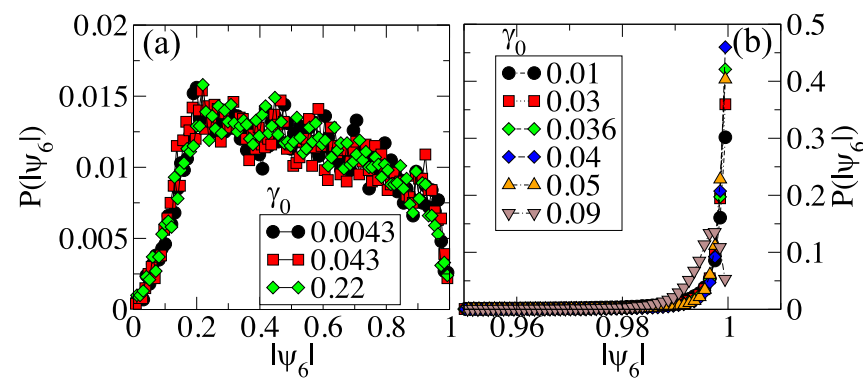

FIG. 6. Probability of hexatic order parameter for different values of strain amplitude for (a) amorphous solid and (b) polycrystalline system at $T=0.001$.

solid, whereas in the polycrystalline system particles with five and seven neighbors (dislocations) mainly appear in grain boundaries. The right panel [Figs. 5(b) and 5(d)] exhibits the configurations of an amorphous and a polycrystalline system, respectively, after the deformation by 200 cycles with a high value of strain amplitude. In the case of amorphous systems, the number of neighbors remains the same while for polycrystalline systems, dislocations (particles with five and seven neighbors) disappear via the atomic rearrangement, and a more ordered crystalline structure is observed. Defect annihilation is also observed in submicrometer dimensional metal crystals [44] and colloidal polycrystals [20] via cyclic loading.

To probe the dynamical heterogeneity, we have shown a color map that illustrates the displacement of particles for both systems at two different strain amplitudes. Figures 7(a) and 7(b) correspond to the displacement of particles in the amorphous solid at strain amplitudes $\gamma_{0}=0.011$ and $\gamma_{0}=$ 0.06 at $T=0.001$. Figures 7(c) and 7(d) represent the same quantity for polycrystalline systems at $\gamma_{0}=0.01$ and $\gamma_{0}=$ 0.06 at $T=0.001$. By a visual inspection, we can comment that displacement fields for a polycrystalline system are much more homogeneous as compared to those of an amorphous system at higher strain amplitude. To quantify, we have plotted probability density of displacement for both cases in Figs. 7(e) and 7(f). Figure 7(e) describes that at a low value of strain amplitude, both exhibit a similar distribution whereas, at higher strain amplitude, the distribution is flat as compared to amorphous solid. Less inhomogeneity in the polycrystalline sample is exhibited through the displacement distribution.

\section{Atomic strain}

To understand the rearrangement in both systems, we look at the Von Mises shear stress, $\eta_{i}^{\text {mises }}$, at particle level [45]. The following algorithm has been used: the initial configuration is considered as reference and the local transformation matrix $\mathbf{J}_{i}$ that best maps $\left\{\mathbf{d}_{j i}^{0}\right\} \rightarrow\left\{\mathbf{d}_{j i}\right\}, \forall j \in P_{i}^{0}$ is formed, where d's are vector separations (row vectors) between atom $j$ and $i$ (superscript 0 means the reference configuration). Here, $j$ is one of atom $i$ 's nearest neighbors, and $P_{i}^{0}$ is the total number of nearest neighbors of atom $i$, at the reference configurations. $\mathbf{J}_{i}$ is determined by minimising $\sum_{j \in P_{i}^{0}}\left|\mathbf{d}_{j i}^{0} \mathbf{J}_{i}-\mathbf{d}_{j i}\right|^{2} \rightarrow \mathbf{J}_{i}$.

For each $\mathbf{J}_{i}$, the local Lagrangian strain matrix is computed as $\eta_{i}=\frac{1}{2}\left(\mathbf{J}_{i} \mathbf{J}_{i}^{T}-\mathbf{I}\right)$. Then the local shear invariant is
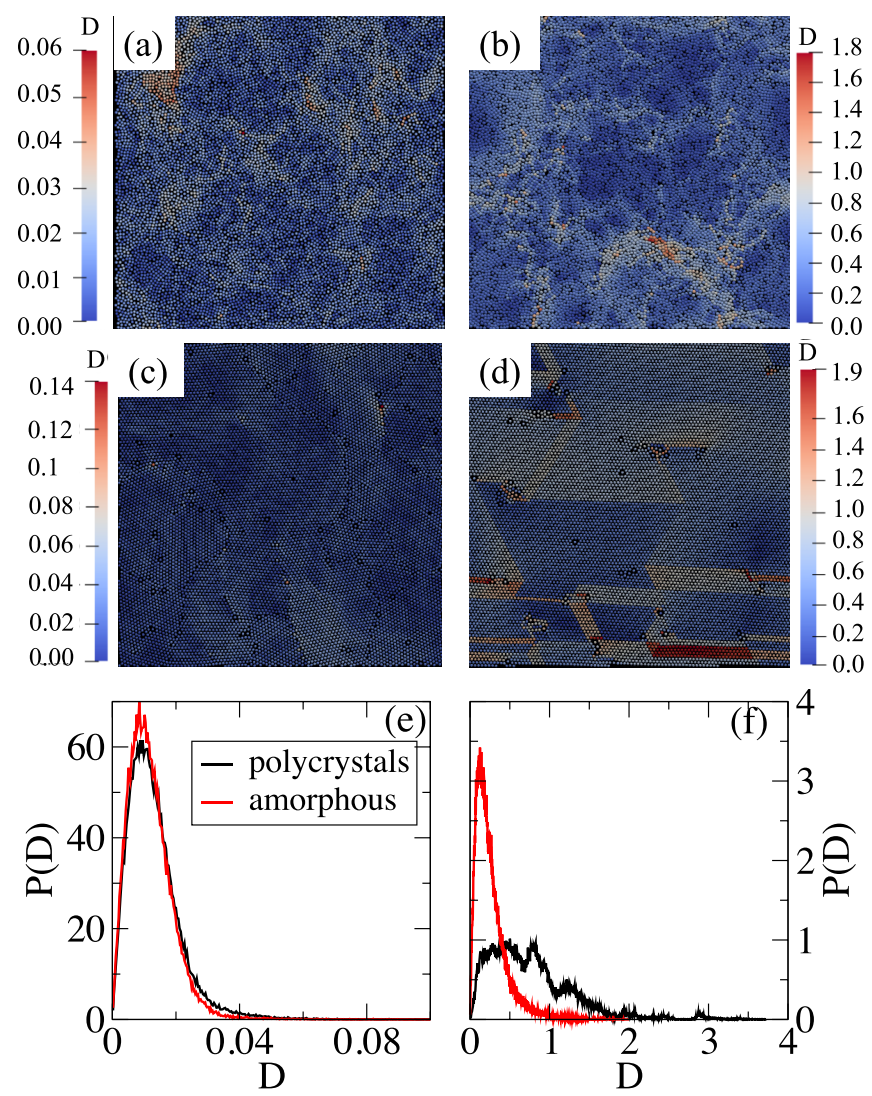

FIG. 7. Displacement magnitude map. Panels (a), (b) show the magnitude of displacement of the particles in amorphous solid during the last strain cycle with a low, $\gamma_{0}=0.011$ and a high value of strain amplitude, $\gamma_{0}=0.06$, respectively. Panels (c), (d) depict the same parameter for the polycrystalline system when $\gamma_{0}$ is 0.01 and 0.06 , respectively. Panels (e), (f) exhibit the probability density distribution of displacement for both systems. Strain amplitudes in (e) correspond to (a) and (c), whereas the strain amplitude in (f) corresponds to the snapshots (b) and (d). All are at temperature $T=0.001$.

calculated for each atom $i$ as

$$
\eta_{i}^{\text {mises }}=\sqrt{\eta_{x y}^{2}+\frac{\left(\eta_{x x}-\eta_{y y}\right)^{2}}{2}}
$$

and shown in Fig. 8.

Figure 8(a) corresponds to amorphous solid and Fig. 8(b) represents the polycrystalline sample. They display $\eta_{\text {mises }}$ for each particle after the application one cycle. We observe local rearrangements in the case of amorphous solid, whereas polycrystalline material exhibits sliding motions of different lengths. The local events in amorphous solid are well identified by Falk and Langer [37] and others [46]. To quantify the sliding motion observed in the polycrystalline sample, we have plotted the probability density $P\left(\eta_{\text {mises }}\right)$ of $\eta_{\text {mises }}$. We see two additional peaks in the distribution in case of the polycrystalline sample as shown in Fig. 8(c). Those peaks correspond to the particles having $0.04<\eta_{\text {mises }}<0.1$ and $0.15<\eta_{\text {mises }}<0.3$, which are denoted as regions I and II, respectively. The arrow in Fig. 8(d) indicates the particles in the regions mentioned above. The mechanism of sliding motion is demonstrated in Fig. 9. In Fig. 9(a), we have shown a part of a polycrystalline sample with grain boundaries 


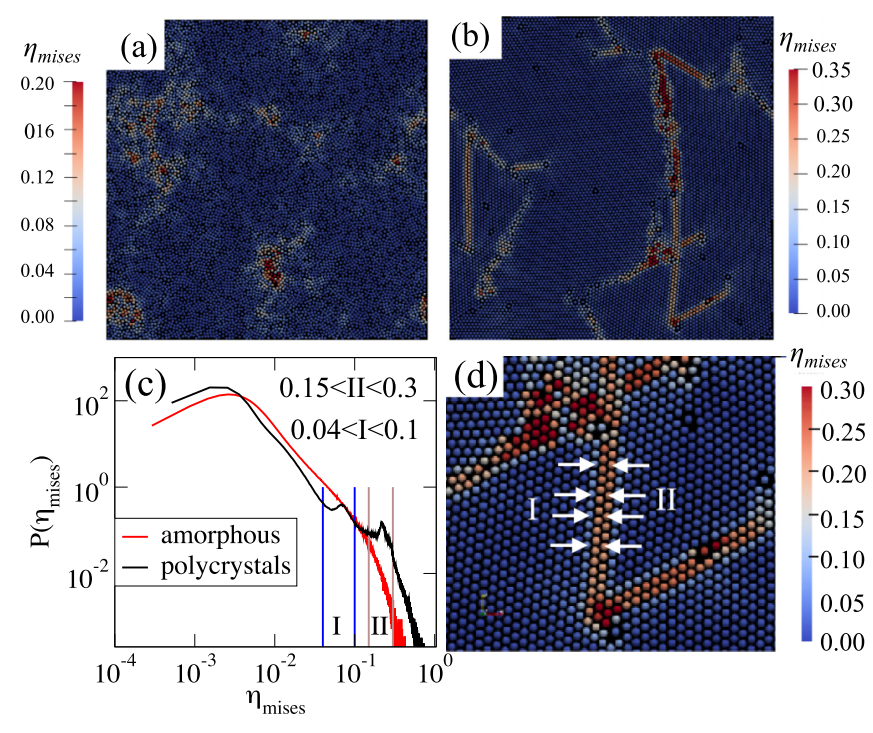

FIG. 8. Atomic strain map. Panels (a), (b) display the atomic strain of each particle with respect to its previous configuration at $\gamma_{0}=0.039$ for amorphous solid and $\gamma_{0}=0.04$ for polycrystalline system, respectively, which are close to the transition. (c) Probability density for von Mises shear strain. Applied strain amplitudes are same as (a) and (b). In case of polycrystalline sample two additional peaks appear in contrast to amorphous solid. (d) A region of snapshot (b) is shown where particles with $\eta_{\text {mises }}$ in the range of the region I and II are indicated by arrows.

(consist of an array of dislocations) and individual dislocations. We focus on the dislocation which is indicated by the yellow circle in Fig. 9(a). Figure 9(b) displays that after one cycle, the dislocation moves from the earlier position. To understand this motion, we compute the displacement field as shown in Fig. 9(c), where it exhibits that two grains are moving opposite to each other. The atomic strain map captures the sliding motion as displayed in Fig. 9(d). From our simulations, we observe that the slip motions are mainly happening along the principal axis of the crystal lattice and have a preference whether the principal axis of the local crystalline structure is along $x$ or $y$ direction. To explain the behavior we consider a
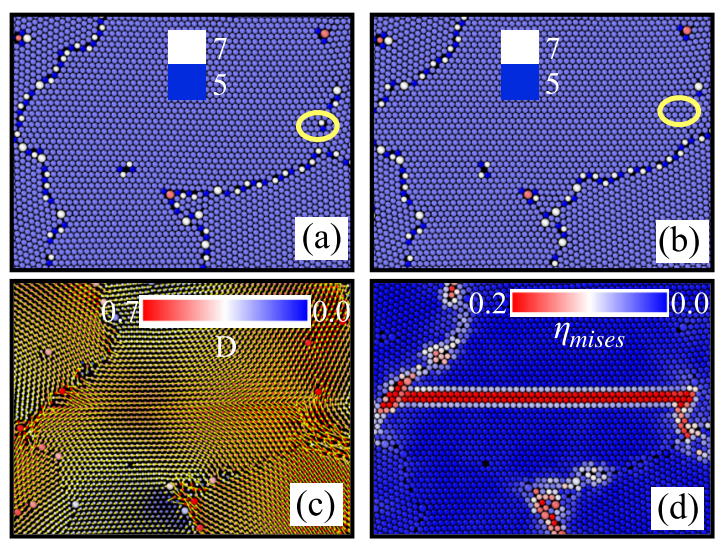

FIG. 9. (a) A region of the polycrystalline sample with grain boundary is shown. (b) Dislocation moves from the location pointed by the yellow circle. (c) Displacement field after the completion of a cycle. (d) von Mises shear strain. similar formalism as performed by Shiba et al. [24] where they studied the polycrystalline sample under steady shear. Using the Peach-Koehler theory one may obtain the elastic energy of the slip in isotropic elasticity as follows [47]:

$$
f_{\text {slip }}=\frac{\mu b^{2}}{2 \pi} \frac{\ln (l / b)}{1-v} \pm \Sigma_{x y} b l \cos (2 \theta),
$$

where $\mu$ is the shear modulus, $b$ is the lattice constant, $v$ is the Poisson's ratio, $l$ is the slip length, $\Sigma_{x y}$ is the external shear deformation, and $\theta$ is the angle made by the slip direction with the $x$ axis. The negative sign implies that particles' displacement around the slip is clockwise and the positive sign implies that particles' displacement around the slip is counterclockwise. The elastic energy of the dislocation is equal to the first term of the equation and the second term is the work done by the applied force. Now, $f_{\text {slip }}$ will be minimum for clockwise movement of the particles $(-\operatorname{sign})$ when $\theta=0$, i.e., the slip is along the $x$ axis and for counterclockwise movement $(+\operatorname{sign})$ when $\theta=\pi / 2$, i.e., the slip is along the $y$ axis. In Fig. 9(c), we have shown that the slip is along the principal axis, and it happens when the crystal is along the $x$ direction. The motion of the particles is clockwise. Shiba et al. have observed similar behavior in a polycrystalline sample [24]. Qualitatively one can comment on the dislocation density from local shear invariant. Probability density of $\eta_{\text {mises }}$ of a system with lower dislocation density decays faster as compared to the system with a larger dislocation density under the same strain amplitude (data are not shown).

In recent investigations, "soft spot" in amorphous solid $[41,48,49]$, which is equivalent to the dislocation in crystalline materials, is identified. Those soft spots are vulnerable toward the rearrangement as compared to other regions in the system. Rottler et al. have performed a detailed comparison between polycrystals and amorphous solid by using the low-frequency quasilocalized modes to identify defects or soft spot [48] that are more likely to fail during plastic deformation [50].

\section{E. Effect of oscillation frequency on irreversible transition}

In addition, we also look at the effect of oscillation frequencies on the irreversibility transition by computing the dynamic modulus. This shows a weak dependence on the transition as shown in Fig. 10. The amorphous solid and the polycrystalline system respond in a similar way. Carrier et al. have observed similar results where they have studied the colloidal glasses under nonlinear oscillatory measurements. They have shown that at a high volume fraction, varying the frequencies by two orders of magnitudes the dynamic moduli weakly changes while at a lower volume fraction the system exhibits a significant response depending on the frequencies [51]. Datta et al. have shown that for the densely packed system, in the linear regime storage modulus $G^{\prime}$ and loss modulus $G^{\prime \prime}$ hardly depend on the oscillation frequencies [52,53]. In a dense granular system, particles are trapped in a "cage," i.e., formed by their neighbors. The mechanism for energy storage and dissipation is guided by the changes in the configuration of these cages and contribute to the moduli. So, the frequency dependence is controlled by the evolution of these cages [54]. In the case of both systems, for different oscillation frequencies, we always see the decrease of storage 


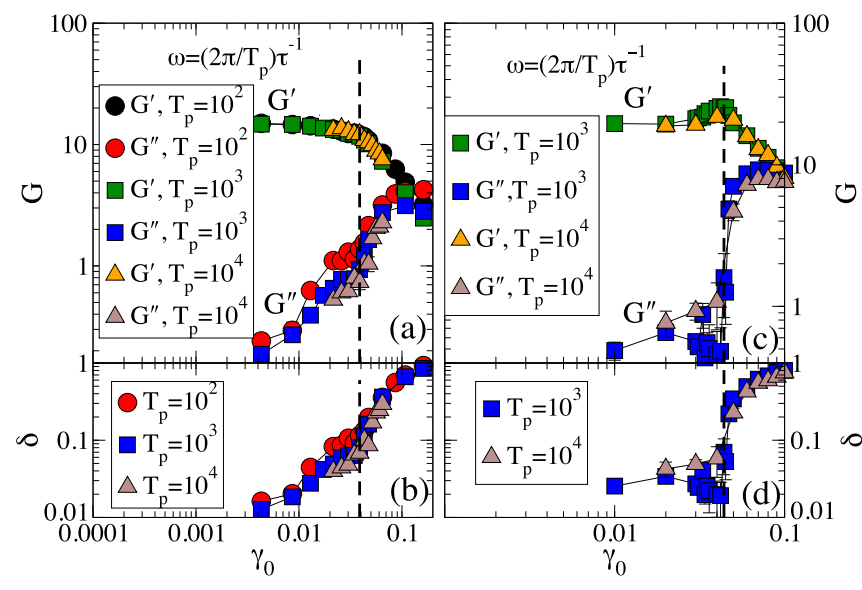

FIG. 10. (a), (c) Dynamic modulus $\left(G^{\prime}, G^{\prime \prime}\right)$ and (b), (d) phase lag $\delta$ are shown as a function of $\gamma_{0}$ for different oscillation frequencies at $T=0.001$. Left panel corresponds to amorphous solid and the right panel is for polycrystalline system.

and the magnitude of shear modulus, $\left|G^{*}\right|=\sqrt{\left(G^{\prime 2}+G^{\prime \prime 2}\right)}$ (not shown here), after a threshold value of strain amplitude which implies the modulus softening of the system. Similar behavior is observed by Lieou et al. [49].

\section{F. Effect of temperature on irreversible transition}

So far, in all previous discussions we have focused on irreversible transition for both amorphous and polycrystalline system at a sufficiently low temperature where thermal fluctuations have hardly any impact on the dynamics of the system within the range of simulation timescale. However, to shed light on the effect of temperatures on the irreversible transition, we also consider a range of temperatures after which amorphous solids exhibit enhanced mobility, and polycrystalline samples annihilate their dislocations rapidly. In Fig. 11(a), we have shown the local slope of mean square displacement $\left(\left\langle\Delta r^{2}\right\rangle\right)$ versus $N_{t} \equiv t / T_{p}$ plot, where $T_{p}=$ $1000 \tau$ (not shown) for amorphous solid at different values of temperatures. We observe that when $T>0.22$ the system exhibits diffusive behavior. In Fig. 11(b), we have shown the number of disclinations (particles with either five or seven neighbors) $N_{\text {dis }}$ as a function of temperature $T$, and we see a rapid decrease when $T>0.1$. Therefore, we apply cyclic load on amorphous solids at four different temperatures $T=$ $0.001,0.01,0.1,0.2$ and on polycrystalline sample at three different temperatures $T=0.001,0.01,0.1$. We compute the average displacement of the particles at the steady state, which is closely similar to the Lindemann criterion where atomic vibration is considered as a precursor to melting [55] in crystals. However, some previous experiments and numerical studies show that the Lindemann criterion can also be valid in glass-liquid transition [56-59]. Lindemann criterion holds universally for the $\alpha$-relaxation irrespective of whether temperature induces the system in the linear response regime or driven by mechanical strain [55]. Our simulations consider both temperature and strain together. We maintain the temperature at a target value and apply different values of strain amplitudes over 200 cycles. We see a competing nature of the applied strain and temperature.

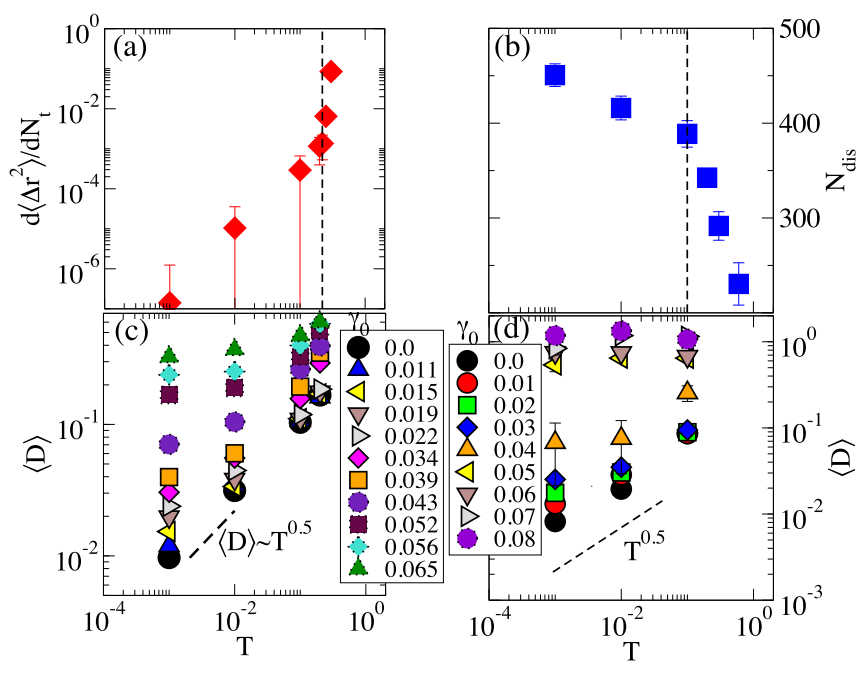

FIG. 11. (a) Local slope of MSD vs. $N_{t} \equiv t / T_{p}$, where $T_{p}=$ $1000 \tau$ for amorphous solid as a function of temperature for amorphous solid. (b) Number of disclinations as a function of temperature for polycrystalline system. The dotted line corresponds to the temperature below which we consider to study the thermal effect. Bottom panel: average displacement of the particles as a function of temperature for different values of strain amplitude. Panel (c) is for amorphous solid and (d) corresponds to a polycrystalline system.

When we look at the average displacement in both cases, we see a clear transition at lower temperatures for both cases; however, it becomes less clear as the temperature increases; see Fig. 11. Our results show that when $\gamma_{0}=0$, average displacement scales as $\langle D\rangle \sim T^{0.5}$ for both cases. However, as we increase the $\gamma_{0}$, the system deviates from the powerlaw behavior. At low temperatures for amorphous systems there is a wide range of displacements depending on strain amplitudes, whereas for polycrystalline systems the case is such that before and after the transition one notices a clear difference.

\section{CONCLUSIONS}

In the present study, by using molecular dynamics simulations we compare the amorphous solid and polycrystalline systems under oscillatory shear. In both cases, we observe enhanced mobility of particles after a threshold value of strain amplitude is reached. The dynamic modulus varies with the amplitude in the same way in both cases. However, the irreversible transition is sharper in the case of polycrystalline sample as compared to the amorphous system.

We observe a clear structural change close to transition in polycrystalline systems, whereas for amorphous solids we see a weak change in the radial distribution function. After the threshold strain amplitude, both systems get fluidized. In addition to that, the distribution of particle displacements in polycrystalline samples is shown to be more homogeneous after the transition as compared to amorphous solids, though at the well below the threshold strain amplitude, displacement distribution is comparable. Finally, we compare the atomic strain for both samples. Polycrystalline systems exhibit a clear signature of sliding dislocations and grain boundary motion which finally leads to the annihilation of defects whereas 
in amorphous systems we see instead local rearrangements. Our simulation, as observed in experiments with the dense colloidal system, shows that oscillation frequency weakly affects the irreversible transition.

To understand the thermal effect on irreversible transition, we apply cyclic deformation on both systems at different temperatures which are below the temperature after which amorphous solid exhibits enhanced mobility and polycrystals annihilate the dislocations sharply. To characterize the transition, we compute the average displacement of the particles. Our simulations show that as temperature increases the irreversibility transition becomes less clear.

A different perspective, not explored here, to better understand the microscopic behavior during yielding in amorphous solids in relation to crystal plasticity relies on the identification of effective defects or soft spots, as discussed in Refs. [48,50].

\section{ACKNOWLEDGMENTS}

P.K.J. and M.J.A. are supported by the Academy of Finland through Project No. 251748 (Centres of Excellence program) and acknowledge the computational resources provided by the Aalto University School of Science Science-IT project. P.K.J. also thanks the Fonds de la Recherche Scientifique de Belgique-FNRS for financial support under Grant No. MIS F.4534.17. S.Z. acknowledges support from the Academy of Finland FiDiPro program, Project No. 13282993 and the European Research Council Advanced Grant No. 291002.
[1] G. Petekidis, A. Moussaid, and P. N. Pusey, Rearrangements in hard-sphere glasses under oscillatory shear strain, Phys. Rev. E 66, 051402 (2002).

[2] S. Slotterback, M. Mailman, K. Ronaszegi, M. van Hecke, M. Girvan, and W. Losert, Onset of irreversibility in cyclic shear of granular packings, Phys. Rev. E 85, 021309 (2012).

[3] R. Mobius and C. Heussinger, (Ir)reversibility in dense granular systems driven by oscillating forces, Soft Matter 10, 4806 (2014).

[4] J. R. Royer and P. M. Chaikin, Precisely cyclic sand: Selforganization of periodically sheared frictional grains, Proc. Natl. Acad. Sci. USA 112, 49 (2015).

[5] L. Corte, P. M. Chaikin, J. P. Gollub, and D. J. Pine, Random organization in periodically driven systems, Nat. Phys. 4, 420 (2008).

[6] E. Tjhung and L. Berthier, Hyperuniform Density Fluctuations and Diverging Dynamic Correlations in Periodically Driven Colloidal Suspensions, Phys. Rev. Lett. 114, 148301 (2015).

[7] T. H. Besseling, M. Hermes, A. Fortini, M. Dijkstra, A. Imhof, and A. van Blaaderen, Oscillatory shear-induced 3D crystalline order in colloidal hard-sphere fluids, Soft Matter 8, 6931 (2012).

[8] M. D. Haw, W. C. K. Poon, and P. N. Pusey, Direct observation of oscillatory-shear-induced order in colloidal suspensions, Phys. Rev. E 57, 6859 (1998).

[9] P. A. Smith, G. Petekidis, S. U. Egelhaaf, and W. C. K. Poon, Yielding and crystallization of colloidal gels under oscillatory shear, Phys. Rev. E 76, 041402 (2007).

[10] D. J. Pine, J. P. Gollub, J. F. Brady, and A. M. Leshansky, Chaos and threshold for irreversibility in sheared suspensions, Nature 438, 997 (2005).

[11] G. I. Menon and S. Ramaswamy, Universality class of the reversible-irreversible transition in sheared suspensions, Phys. Rev. E 79, 061108 (2009).

[12] P. Leishangthem, A. D. S. Parmar, and S. Sastry, The yielding transition in amorphous solids under oscillatory shear deformation, Nat. Commun. 8, 14653 (2017).

[13] N. V. Priezjev, The yielding transition in periodically sheared binary glasses at finite temperature, Comput. Mater. Sci. 150, 162 (2018).

[14] N. C. Keim and P. E. Arratia, Yielding and microstructure in a 2D jammed material under shear deformation, Soft Matter $\mathbf{9}$, 6222 (2013).
[15] L. Pérez-Ocampo, A. Zaccone, and M. Laurati, A well defined glass state obtained by oscillatory shear, J. Rheol. 62, 197 (2018).

[16] M. Blank-Burian and A. Heuer, Shearing small glass-forming systems: A potential energy landscape perspective, Phys. Rev. E 98, 033002 (2018).

[17] E. D. Knowlton, D. J. Pine, and L. Cipelletti, A microscopic view of the yielding transition in concentrated emulsions, Soft Matter 10, 6931 (2014).

[18] S. Dagois-Bohy, E. Somfai, B. P. Tighe, and M. van Hecke, Softening and yielding of soft glassy materials, Soft matter 13, 9036 (2017).

[19] T. Kawasaki and L. Berthier, Macroscopic yielding in jammed solids is accompanied by a nonequilibrium first-order transition in particle trajectories, Phys. Rev. E 94, 022615 (2016).

[20] I. Buttinoni, M. Steinacher, H. Th. Spanke, J. Pokki, S. Bahmann, B. Nelson, G. Foffi, and L. Isa, Colloidal polycrystalline monolayers under oscillatory shear, Phys. Rev. E 95, 012610 (2017).

[21] E. Tamborini, L. Cipelletti, and L. Ramos, Plasticity of a Colloidal Polycrystal Under Cyclic Shear, Phys. Rev. Lett. 113, 078301 (2014).

[22] B. van der Meer, W. Qi, J. Sprakel, L. Filion, and M. Dijkstra, Dynamical heterogeneities and defects in two-dimensional soft colloidal crystals, Soft Matter 11, 9385 (2015).

[23] P. Kumar Jana, M. J. Alava, and S. Zapperi, Irreversibility transition of colloidal polycrystals under cyclic deformation, Sci. Rep. 7, 45550 (2017).

[24] H. Shiba and A. Onuki, Plastic deformations in crystal, polycrystal, and glass in binary mixtures under shear: Collective yielding, Phys. Rev. E 81, 051501 (2010).

[25] N. Koumakis, J. F. Brady, and G. Petekidis, Amorphous and ordered states of concentrated hard spheres under oscillatory shear, J. Non-Newtonian Fluid Mech. 233, 119 (2016).

[26] R. Brüning, D. A. St-Onge, S. Patterson, and W. Kob, Glass transitions in one-, two-, three-, and four-dimensional binary lennard-jones systems, J. Phys.: Condens. Matter 21, 035117 (2008).

[27] G. Drazer, J. Koplik, A. Acrivos, and B. Khusid, Adsorption Phenomena in the Transport of a Colloidal Particle Through a Nanochannel Containing a Partially Wetting Fluid, Phys. Rev. Lett. 89, 244501 (2002). 
[28] C. Negri, A. L. Sellerio, S. Zapperi, and M. Carmen Miguel, Deformation and failure of curved colloidal crystal shells, Proc. Natl. Acad. Sci. USA 112, 14545 (2015).

[29] W. T. Read and W. Shockley, Dislocation models of crystal grain boundaries, Phys. Rev. 78, 275 (1950).

[30] F. van Swol and D. N. Petsev, Molecular dynamics simulation of binary hard sphere colloids near the glass transition, RSC Advances 4, 21631 (2014).

[31] E. Zaccarelli, G. Foffi, K. A. Dawson, S. V. Buldyrev, F. Sciortino, and P. Tartaglia, Confirmation of anomalous dynamical arrest in attractive colloids: A molecular dynamics study, Phys. Rev. E 66, 041402 (2002).

[32] M. J. Stevens, M. O. Robbins, and J. F. Belak, Shear Melting of Colloids: A Nonequilibrium Phase Diagram, Phys. Rev. Lett. 66, 3004 (1991).

[33] H. Komatsugawa and S. Nosé, Nonequilibrium molecular dynamics simulations of oscillatory sliding motion in a colloidal suspension system, Phys. Rev. E 51, 5944 (1995).

[34] B. J. Ackerson and P. N. Pusey, Shear-Induced Order in Suspensions of Hard Spheres, Phys. Rev. Lett. 61, 1033 (1988).

[35] S. Plimpton, Fast parallel algorithms for short-range molecular dynamics, J. Comput. Phys. 117, 1 (1995).

[36] I. Regev, J. Weber, C. Reichhardt, K. A. Dahmen, and T. Lookman, Reversibility and criticality in amorphous solids, Nat. Commun. 6, 11 (2015).

[37] M. L. Falk and J. S. Langer, Dynamics of viscoplastic deformation in amorphous solid, Phys. Rev. E 57, 7192 (1998).

[38] K. K. Chawla and M. A. Meyers, Mechanical Behavior of Materials (Prentice Hall, New York, 1999).

[39] Y. Cohen, S. Karmakar, I. Procaccia, and K. Samwer, The nature of the $\beta$-peak in the loss modulus of amorphous solids, Europhys. Lett. 100, 36003 (2012).

[40] L. Laurson and M. J. Alava, Dynamic Hysteresis in Cyclic Deformation of Crystalline Solids, Phys. Rev. Lett. 109, 155504 (2012).

[41] N. Perchikov and E. Bouchbinder, Variable-amplitude oscillatory shear response of amorphous materials, Phys. Rev. E 89, 062307 (2014).

[42] Y. L. Wu, D. Derks, A. van Blaaderen, and A. Imhof, Melting and crystallization of colloidal hard-sphere suspensions under shear, Proc. Natl. Acad. Sci. USA 106, 10564 (2009).

[43] K. H. Nagamanasa, S. Gokhale, R. Ganapathy, and A. K. Sood, Confined glassy dynamics at grain boundaries in colloidal crystals, Proc. Natl. Acad. Sci. USA 108, 11323 (2011).

[44] Z.-J. Wang, Q.-J. Li, Y.-N. Cui, Z.-L. Liu, E. Ma, J. Li, J. Sun, Z. Zhuang, M. Dao, Z.-W. Shan et al., Cyclic deformation leads to defect healing and strengthening of small-volume metal crystals, Proc. Natl. Acad. Sci. USA 112, 13502 (2015).
[45] S. Futoshi, O. Shigenobu, and J. Li, Theory of shear banding in metallic glasses and molecular dynamics calculations, Mater. Trans. 48, 2923 (2007).

[46] A. Tanguy, F. Leonforte, and J.-L. Barrat, Plastic response of a 2D Lennard-Jones amorphous solid: Detailed analysis of the local rearrangements at very slow strain rate, Eur. Phys. J. E 20, 355 (2006).

[47] M. Peach and J. S. Koehler, The forces exerted on dislocations and the stress fields produced by them, Phys. Rev. 80, 436 (1950).

[48] M. L. Manning and A. J. Liu, Vibrational Modes Identify Soft Spots in a Sheared Disordered Packing, Phys. Rev. Lett. 107, 108302 (2011).

[49] C. K. C. Lieou, E. G. Daub, R. A. Guyer, and P. A. Johnson, Nonlinear softening of unconsolidated granular earth materials, J. Geophys. Res.: Solid Earth 122, 6998 (2017).

[50] J. Rottler, S. S. Schoenholz, and A. J. Liu, Predicting plasticity with soft vibrational modes: From dislocations to glasses, Phys. Rev. E 89, 042304 (2014).

[51] V. Carrier and G. Petekidis, Nonlinear rheology of colloidal glasses of soft thermosensitive microgel particles, J. Rheol. 53, 245 (2009).

[52] S. S. Datta, D. D. Gerrard, T. S. Rhodes, T. G. Mason, and D. A. Weitz, Rheology of attractive emulsions, Phys. Rev. E 84, 041404 (2011).

[53] T. G. Mason, New fundamental concepts in emulsion rheology, Curr. Opin. Colloid Interface Sci. 4, 231 (1999).

[54] T. G. Mason and D. A. Weitz, Linear Viscoelasticity of Colloidal Hard Sphere Suspensions Near the Glass Transition, Phys. Rev. Lett. 75, 2770 (1995).

[55] H.-B. Yu, R. Richert, R. Maaß, and K. Samwer, Unified Criterion for Temperature-Induced and Strain-Driven Glass Transitions in Metallic Glass, Phys. Rev. Lett. 115, 135701 (2015).

[56] L. Larini, A. Ottochian, C. De Michele, and D. Leporini, Universal scaling between structural relaxation and vibrational dynamics in glass-forming liquids and polymers, Nat. Phys. 4, 42 (2008).

[57] A. Ottochian, C. De Michele, and D. Leporini, Universal divergenceless scaling between structural relaxation and caged dynamics in glass-forming systems, J. Chem. Phys. 131, 224517 (2009).

[58] W. H. Wang, P. Wen, D. Q. Zhao, M. X. Pan, and R. J. Wang, Relationship between glass transition temperature and Debye temperature in bulk metallic glasses, J. Mater. Res. 18, 2747 (2003).

[59] X. Xia and P. G. Wolynes, Fragilities of liquids predicted from the random first order transition theory of glasses, Proc. Natl. Acad. Sci. USA 97, 2990 (2000). 